\title{
NOTE ON THE PROJECTIONS OF THE ABSOLUTE ACCELERATION IN RELATIVE MOTION.
}

BY DR. G. O. JAMES.

INASMUCH as the usual methods of forming the projections of the absolute acceleration on the moving axes rest to a certain extent on geometric considerations it has seemed worth while to treat the question from a purely analytic point of view and derive these expressions by purely algebraic processes. This method possesses the advantage of being immediately extensible to any number of dimensions, and in the latter part of this note $I$ shall give the expressions for $n$ dimensions.

Connecting the moving axes $O x, O y, O z$ with the fixed axes by the following schema

$$
\begin{array}{c|ccc}
\multicolumn{1}{c}{} & x & y & z \\
\hline X & a & b & c \\
Y & \alpha^{\prime} & b^{\prime} & c^{\prime} \\
Z & a^{\prime \prime} & b^{\prime \prime} & c^{\prime \prime},
\end{array}
$$

I shall rapidly recall the following equations :

$$
X=S a x, \quad Y=S \alpha^{\prime} x, \quad Z=S \alpha^{\prime \prime} x,
$$

where $S$ denotes summation with respect to $a, b, c$ and $x, y, z$.

$$
\left\{\begin{array}{l}
\frac{d X}{d t}=S a \frac{d x}{d t}+S x \frac{d a}{d t}, \\
\frac{d Y}{d t}=S a^{\prime} \frac{d x}{d t}+S x \frac{d a^{\prime}}{d t}, \\
\frac{d Z}{d t}=S a^{\prime \prime} \frac{d x}{d t}+S x \frac{d a^{\prime \prime}}{d t}
\end{array}\right.
$$


(3)

$$
\begin{aligned}
& \left\{\begin{array}{l}
\frac{d^{2} X}{d t^{2}}=S a \frac{d^{2} x}{d t^{2}}+S x \frac{d^{2} a}{d t^{2}}+2 S \frac{d x}{d t} \frac{d a}{d t}, \\
\frac{d^{2} Y}{d t^{2}}=S a^{\prime} \frac{d^{2} x}{d t^{2}}+S x \frac{d^{2} a^{\prime}}{d t^{2}}+2 S \frac{d x}{d t} \frac{d a^{\prime}}{d t}, \\
\frac{d^{2} Z}{d t^{2}}=S a^{\prime \prime} \frac{d^{2} x}{d t^{2}}+S x \frac{d^{2} a^{\prime \prime}}{d t^{2}}+2 S \frac{d x}{d t} \frac{d a^{\prime \prime}}{d t}
\end{array} ;\right. \\
& \left\{\begin{array}{l}
p=\sum c \frac{d b}{d t}=-\sum b \frac{d c}{d t} \\
q=\sum a \frac{d c}{d t}=-\sum c \frac{d a}{d t} \\
r=\sum b \frac{d a}{d t}=-\sum a \frac{d b}{d t} .
\end{array}\right.
\end{aligned}
$$

The projections of the absolute velocity on the moving axes are then

$$
\left\{\begin{array}{l}
V_{x}=\sum a \frac{d X}{d t}=\frac{d x}{d t}+q z-r y \\
V_{y}=\sum b \frac{d X}{d t}=\frac{d y}{d t}+r x-p z \\
V_{z}=\sum c \frac{d X}{d t}=\frac{d z}{d t}+p y-q x
\end{array}\right.
$$

The direction cosines satisfy the following equations

$$
\frac{d a}{d t}=b r-c q, \quad \frac{d b}{d t}=c p-a r, \quad \frac{d c}{d t}=a q-b p,
$$

with two similar sets in $a^{\prime}, b^{\prime}, c^{\prime}$, and $a^{\prime \prime}, b^{\prime \prime}, c^{\prime \prime}$, which I shall denote by $(5)^{\prime}$ and $(5)^{\prime \prime}$.

The projections of the absolute acceleration on the moving axes are

$$
(6)\left\{\begin{array}{l}
J_{x}=\sum a \frac{d^{2} X}{d t^{2}}=\sum a S \alpha \frac{d^{2} x}{d t^{2}}+\sum a S x \frac{d^{2} a}{d t^{2}}+2 \sum a S \frac{d x}{d t} \frac{d a}{d t}, \\
J_{y}=\sum b \frac{d^{2} X}{d t^{2}}=\ldots, \\
J_{z}=\sum c \frac{d^{2} X}{d t^{2}}=\ldots .
\end{array}\right.
$$


Calculating $\sum a S a \frac{d^{2} x}{d t^{2}}$ and remembering the relations between the direction cosines we have at once

$$
\sum a S a \frac{d^{2} x}{d t^{2}}=\frac{d^{2} x}{d t^{2}}
$$

Again

$$
\sum a S x \frac{d^{2} a}{d t^{2}}=x \sum a \frac{d^{2} a}{d t^{2}}+y \sum a \frac{d^{2} b}{d t^{2}}+z \sum a \frac{d^{2} c}{d t^{2}} .
$$

Differentiating (5) (5) and (5)" and calculating

$$
\sum a \frac{d^{2} a}{d t^{2}}, \quad \sum a \frac{d^{2} b}{d t^{2}}, \quad \sum a \frac{d^{2} c}{d t^{2}},
$$

we have

$$
\left\{\begin{array}{l}
\sum a \frac{d^{2} a}{d t^{2}}=-\left(q^{2}+r^{2}\right) \\
\sum a \frac{d^{2} b}{d t^{2}}=-\frac{d r}{d t}+p q \\
\sum a \frac{d^{2} c}{d t^{2}}=\frac{d q}{d t}+p r
\end{array}\right.
$$

Again

$$
\sum a S \frac{d x}{d t} \frac{d a}{d t}=\frac{d x}{d t} \sum a \frac{d a}{d t}+\frac{d y}{d t} \sum a \frac{d b}{d t}+\frac{d z}{d t} \sum a \frac{d c}{d t}
$$

Hence

$$
=-r \frac{d y}{d t}+q \frac{d z}{d t} \text {. }
$$

$$
J_{x}=\frac{d^{2} x}{d t^{2}}-\left(q^{2}+r^{2}\right) x+\left(p q-\frac{d r}{d t}\right) y+\left(p r+\frac{d q}{d t}\right) z
$$

Now (10) may be written

$$
-12\left(r \frac{d y}{d t}-q \frac{d z}{d t}\right) *
$$

$$
\begin{aligned}
J_{x}=\frac{d}{d t}\left(\frac{d x}{d t}+q z-r y\right)+q\left(\frac{d z}{d t}\right. & +p y-q x) \\
& -r\left(\frac{d y}{d t}+r x-p z\right) .
\end{aligned}
$$

* This formula appears to have been first given by J. Liouville, Jour. de Math. pures et appl., series 2, vol. 3 (1858), pp. 10-11. 
Hence we have for the projections the ordinary expressions

$$
\left\{\begin{array}{l}
J_{x}=\frac{d V_{x}}{d t}+q V_{z}-r V_{y}, \\
J_{y}=\frac{d V_{y}}{d t}+r V_{x}-p V_{z}, \\
J_{z}=\frac{d V_{z}}{d t}+p V_{y}-q V_{x} .
\end{array}\right.
$$

To extend this method to $n$ dimensions I shall connect the moving axes $O x_{1}, O x_{2} \cdots, O x_{n}$ with the fixed axes $O X_{a}$, $O X_{\beta}, \cdots, O X_{\nu}$ by the following table :

Then

$$
\begin{array}{c|cccc}
\multicolumn{1}{c}{} & x_{1} & x_{2} & \cdots & x_{n} \\
\cline { 2 - 5 } X_{a} & a_{1} & a_{2} & \cdots & a_{n} \\
X_{\beta} & \beta_{1} & \beta_{2} & \cdots & \beta_{n} \\
\vdots & \vdots & \vdots & \vdots & \vdots \\
X^{n} & & & &
\end{array}
$$

where

$$
\begin{gathered}
X_{\lambda}=\sum_{i=1}^{n} \lambda_{i} x_{i}, \\
\frac{d X_{\lambda}}{d t}=\sum_{i=1}^{n} \lambda_{i} \frac{d x_{i}}{d t}+\sum_{i=1}^{n} x_{i} \frac{d \lambda_{i}}{d t} \\
\frac{d^{2} X_{\lambda}}{d t^{2}}=\sum_{i=1}^{n} \lambda_{i} \frac{d^{2} x_{i}}{d t^{2}}+\sum_{i=1}^{n} x_{i} \frac{d^{2} \lambda_{i}}{d t^{2}}+2 \sum_{1=i}^{n} \frac{d x_{i}}{d t} \frac{d \lambda_{i}}{d t} \\
V_{x_{\tau}}=S a_{\tau} \frac{d X_{a}}{d t}=S a_{\tau} \sum_{i=1}^{n} a_{i} \frac{d x_{i}}{d t}+S a_{\tau} \sum_{i=1}^{n} x_{i} \frac{d a_{i}}{d t} \\
=\sum_{i=1}^{n} S a_{\tau} a_{i} \frac{d x_{i}}{d t}+\sum_{i=1}^{n} S a_{\tau} \frac{d a_{i}}{d t} x_{i} \\
=\frac{d x_{\tau}}{d t}+\sum_{i=1}^{n} x_{i} S a_{\tau} \frac{d a_{i}}{d t}=\frac{d x_{\tau}}{d t}+\sum_{i=1}^{n} x_{i} p_{i \tau},
\end{gathered}
$$


1902.] PRojections of Absolute acceleration.

$$
\begin{gathered}
p_{i \tau} \equiv S a_{\tau} \frac{d a_{i}}{d t}=-S a_{i} \frac{d a_{\tau}}{d t}, \\
J_{x_{\tau}}=S a_{\tau} \frac{d^{2} X_{a}}{d t^{2}}=S a_{\tau} \sum_{i=1}^{n} a_{i} \frac{d^{2} x_{i}}{d t^{2}}+S a_{\tau} \sum_{i=1}^{n} x_{i} \frac{d^{2} a_{i}}{d t^{2}} \\
+2 S a_{\tau} \sum_{i=1}^{n} \frac{d x_{i}}{d t} \frac{d a_{i}}{d t} \\
=\sum_{i=1}^{n} \frac{d^{2} x_{i}}{d t^{2}} S a_{\tau} a_{i}+\sum_{i=1}^{n} x_{i} S a_{\tau} \frac{d^{2} a_{i}}{d t^{2}}+2 \sum_{i=1}^{n} \frac{d x_{i}}{d t} S a_{\tau} \frac{d a_{i}}{d t} .
\end{gathered}
$$

The differential equation satisfied by the direction cosine $\lambda_{i}$ is found by expressing the fact that the point at unit distance on the axis $O X_{\lambda}$ has a velocity zero along the moving axes. Hence

$$
\frac{d \lambda_{i}}{d t}+\sum_{j=1}^{n} \lambda_{j} p_{i i}=0
$$

From (18)

$$
\frac{d^{2} \lambda_{i}}{d t^{2}}=\sum_{j=1}^{n} \lambda_{j} \frac{d p_{i j}}{d t}+\sum_{j=1}^{n} \frac{d \lambda_{j}}{d t} p_{i j}
$$

Whence

(20) $S a_{\tau} \frac{d^{2} a_{i}}{d t^{2}}=\sum_{j=1}^{n} \frac{d p_{i j}}{d t} S a_{\tau} a_{j}+\sum_{j=1}^{n} p_{i j} S a_{\tau} \frac{d a_{i}}{d t}=\frac{d p_{i \tau}}{d t}+\sum_{j=1}^{n} p_{i j} p_{j \tau}$. Hence

(21) $J_{x_{\tau}}=\frac{d^{2} x_{\tau}}{d t^{2}}+\sum_{i=1}^{n} x_{i}\left(\frac{d p_{i \tau}}{d t}+\sum_{j=1}^{n} p_{i j} p_{j \tau}\right)+2 \sum_{i=1}^{n} \frac{d x_{i}}{d t} p_{i \tau}$

$$
\begin{aligned}
& =\frac{d}{d t}\left(\frac{d x_{\tau}}{d t}+\sum_{i=1}^{n} x_{i} p_{i \tau}\right)+\sum_{i=1}^{n}\left(\frac{d x_{i}}{d t}+\sum_{j=1}^{n} x_{j} p_{j i}\right) p_{i \tau} \\
& =\frac{d}{d t} V_{x \tau}+\sum_{i=1}^{n} V_{x_{\tau}} p_{i_{\tau}} \quad(\tau=1,2 \cdots, n) .
\end{aligned}
$$

LeHigh UNiversity. 